\title{
Markierungsfreie optische Detektion von Antibiotika zur in-line Prozessanalytik
}

\author{
P. Weber ${ }^{1}$, B. Riegger ${ }^{2}$, M. Bach ${ }^{2}$, G. Tovar ${ }^{2}$ und G. Gauglitz ${ }^{1}$ \\ ${ }^{1}$ Institut für Physikalische und Theoretische Chemie, Universität Tübingen, Tübingen, Deutschland \\ ${ }^{2}$ Institut für Grenzflächenverfahrenstechnik und Plasmatechnologie, \\ Universität Stuttgart, Stuttgart, Deutschland
}

\begin{abstract}
Zusammenfassung
Im Rahmen des Projektes BioMIP soll ein optischer Sensor zur in-line Prozessanalytik von Fermentationsprozessen entwickelt werden. Dabei ist das Ziel, die Konzentration von Produkten wie z.B. Penicillin $G$ direkt innerhalb eines Fermenters zu bestimmen.

Auf Grund der Gegebenheiten in einem Fermenter werden als Erkennungsstrukturen molekular geprägte Polymerpartikel (MIPs) verwendet. Die Wechselwirkungen zwischen den Polymerpartikeln und den Zielsubstanzen werden hierbei mittels 1-lambda Reflektometrie (1- $\lambda$-RIfS) von außerhalb des Fermenters durch eine Glasscheibe berührungsfrei und zeitaufgelöst ausgelesen. Die Messtechnik ist hierbei so gestaltet, dass verschiedene Messspots (MIP und ungeprägte Polymerpartikel (NIP)) angesteuert und parallel ausgelesen werden können. Dies hat den Vorteil, dass eine interne Referenzierung zwischen den beiden Spots möglich ist und somit unspezifische Signale der Polymerpartikel eliminiert werden können.

Es wurden bereits Strategien zur Immobilisierung der Polymerpartikel entwickelt und reproduzierbare und konzentrationsabhängige Messungen mit solch einem optischen Sensor für den Analyten Penicillin G durchgeführt.
\end{abstract}

Schlagwörter: optischer Chemosensor, Antibiotika, markierungsfrei, Molekular geprägte Moleküle (MIP), inline Analytik

\section{Einleitung}

Fermentationsprozesse sind als biotechnologische Methode für industrielle Produktionsverfahren ein boomender Wirtschaftszweig und haben bereits eine Vielzahl an Anwendungen in der Industrie gefunden. Beispiele für solche Fermentationsprozesse sind die Produktion von Impfstoffen, Feinchemikalien, Arzneimitteln und Zusatzstoffen wie Aminosäuren und Vitamine. Um eine optimale Produktausbeute zu erhalten, müssen bei der Prozessführung die Bedingungen stets an die aktuellen Gegebenheiten angepasst werden. Um dies zu erreichen, muss eine exakte Analytik prozessrelevanter Parameter vorausgesetzt werden. Dabei wird zwischen off-line, at-line und in-line Prozessanalytik unterschieden [1].

Da die Anforderungen an solch ein Sensorsystem durch das aggressive und komplexe Probenmedium sehr hoch sind, muss der entsprechende Sensor eine hohe Robustheit aufweisen, sterilisierbar und relativ inert gegenüber Matrixeffekten des Fermentationsmediums sein. Um dies zu erreichen, kommen molekular geprägte Polymerpartikel (MIP) sowie ungeprägte
Polymerpartikel (NIP) als sensitive Schicht zum Einsatz.

Häufig sind bei solchen Polymerpartikeln, die als Erkennungstrukturen fungieren, unspezifische Wechselwirkungen ein großes Problem. Durch diese unspezifischen Wechselwirkungen ist es oft schwierig die spezifischen Wechselwirkungen des Analyten sicher zu detektieren.

Um dieses Problem zu lösen, ist eine interne Referenzierung unumgänglich. Bei dieser internen Referenzierung werden verschiedene Messspots (MIP und NIP) parallel ausgelesen. Dies ist mit einem 1-lambda Reflektometrie Messgerät mit verschiebbaren $x$-y-Tisch möglich.

\section{Methoden und Materialien}

1-lambda Reflektometrie (1- $\lambda$-RIfS)

Als Detektionsprinzip wurde die $1-\lambda$-RIfS gewählt. Es handelt sich um eine markierungsfreie und zeitaufgelöste Methode. Sie beruht auf dem Prinzip von Mehrfachreflexion an dünnen Schichten [2-5].

Wie in Abb.1.1 zu sehen ist, wird ein transparentes Mehrschichtsystem, bestehend aus 
einem Glastransducer und einer sensitiven Schicht, von der Unterseite mit einer LED $(\lambda=470$ $\mathrm{nm})$ bestrahlt. Die reflektierten Strahlen interferieren und bilden ein charakteristisches Interferenzspektrum. Bindet sich nun wie in Abb.1.2 dargestellt der Analyt (Penicillin G) an die Sensoronerfläche, verursacht dies eine Änderung der optischen Schichtdicken (nd). Daraus resultiert eine Verschiebung des Interferenzspektrums. Schließlich wird ein zeitaufgelöstes Bindungssignal durch Detektion der Änderung der reflektierten Lichtintensität bei einer Wellenlänge erhalten (Abb.1.3).

1

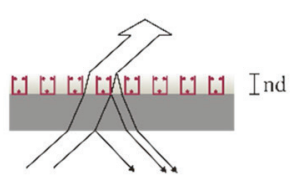

2
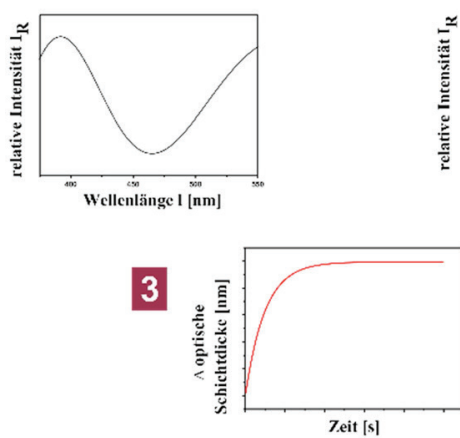

Abb.1: RIfS-Detektionsprinzip: Charakteristisches Interferenzspektrum ohne Anlagerung des Analyten (1). Verschiebung des Charakteristischen Interferenzspektrums durch Anlagerung des Analyten an die Sensoroberfläche (2). Zeitaufgelöstes Bindungssignal (3).

\section{Messaufbau}

Um die unspezifischen Wechselwirkungen der Polymerpartikel zu eliminieren und eine Referenzierung zu gewährleisten, wird ein 2-Spot Messaufbau verwendet. Dieser Messaufbau besitzt einen verschiebbaren $x-y$-Tisch [6].

Durch Verschieben dieses $x-y$-Tisches, der die Kavität mit Flusszelle beinhaltet, können einzelne Messpositionen auf dem Transducer vom darunter befindlichen Detektionsmodul ausgelesen werden. Es wurden auf einem Transducer zwei Spots für MIP und NIP aufgetragen. Somit ist eine Messung von beiden Nanopartikelspots quasiparallel möglich.

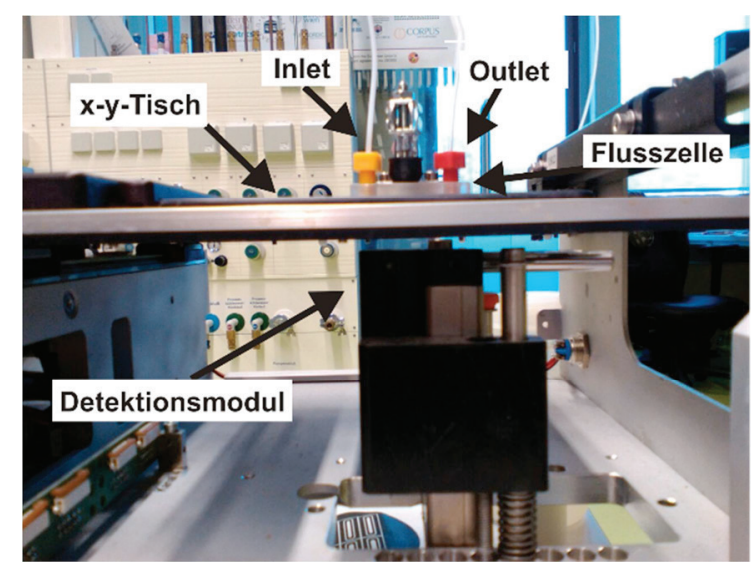

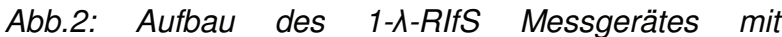
verschiebbaren $x$-y-Tisches.

\section{Molekular geprägte Polymere}

Als sensitive Schicht werden MIP- bzw. NIPPartikel eingesetzt. Zur Herstellung dieser Nanopartikel werden Monomere in Gegenwart von Zielanalyten polymerisiert. Danach wird der Zielanalyt aus dem Polymer extrahiert. Im Polymer verbleiben nun spezifische Bindungstellen für den Zielanalyten. Die Polymerpartikel wurden elektrostatisch durch Carboxypolyethylenglycol, das als Linker fungiert, an der Oberfläche immobilisiert.

\section{Ergebnisse}

Immobilisierung der Polymerpartikel

Um zu überprüfen, ob die Polymerpartikel elektrostatisch an der funktionalisierten Oberfläche gebunden haben, wurden AFM- und REM-Aufnahmen durchgeführt. In Abb.3 ist eine AFM-Aufnahme einer mit MIP-Partikel beschichteten Oberfläche zu sehen. Es ist zu sehen, dass die geprägten Polymerpartikel erfolgreich an der Oberfläche immobilisiert wurden. Allerdings wird auch deutlich, dass die Oberfläche nicht komplett homogen ist und sich immer wieder Agglomerate gebildet haben.

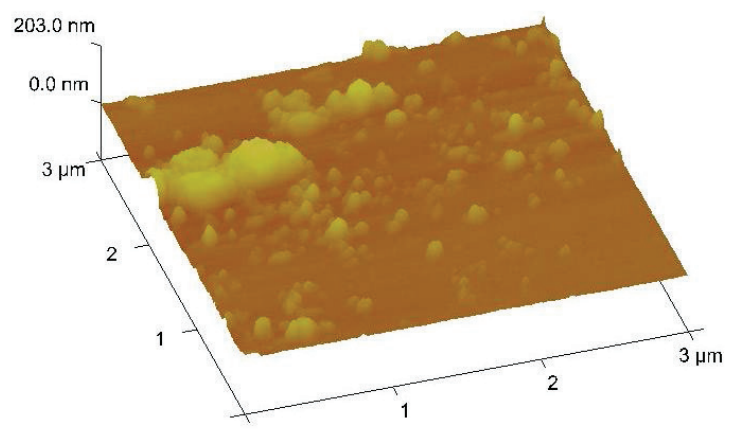

Abb.3: AFM-Aufnahmen eines Glastransducers auf dem elektrostatisch MIP-Partikel immobilisiert wurde. Referenzierung 
Ein wichtiger Punkt in dieser Arbeit ist die parallele Auslesung der beiden Messspots (MIP und NIP). Die Vorraussetzung dafür ist, dass die Sensorsignale der beiden Spots deutlich voneinander zu unterscheiden sind. In Abb. 4 sind Sensonsorsignale der parallelen Auslesung der beiden Spots bei verschiedenen Konzentration zu sehen. Es können eindeutig die MIP- und NIPSignale voneinander unterschiedenen werden.

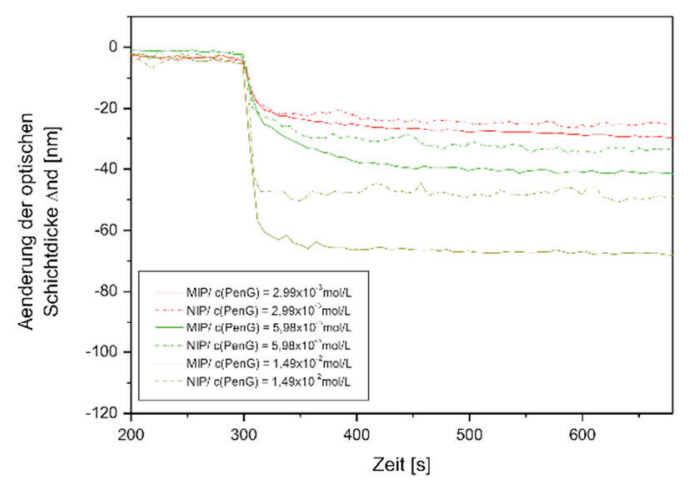

Abb.4: Sensorsignale der parallelen Auslesung des MIP- bzw. NIP-Spots bei verschiedenen Konzentrationen.

\section{Konzentrationsabhängige Messungen}

Dreifachmessungen verschiedener Konzentrationen sind in Abb. 5 gezeigt. Es wurden dabei Konzentrationen im Bereich von $1,49 \cdot 10^{-3} \mathrm{~mol} / \mathrm{L}$ bis $14,9 \cdot 10^{-3} \mathrm{~mol} / \mathrm{L}$ gewählt. In rot ist die Änderung der optischen Schichtdicke bei verschiedenen Konzentrationen für den NIP-Spot und in schwarz für den MIP-Spot zu sehen. Sie können deutlich voneinander unterschieden werden. Auch eine Konzentrationsabhängigkeit ist $\mathrm{zu}$ erkennen. Deutlich wird, dass ab der Konzentration von $5,98 \cdot 10^{-3} \mathrm{~mol} / \mathrm{L}$ ein fast linearer Bereich beginnt.

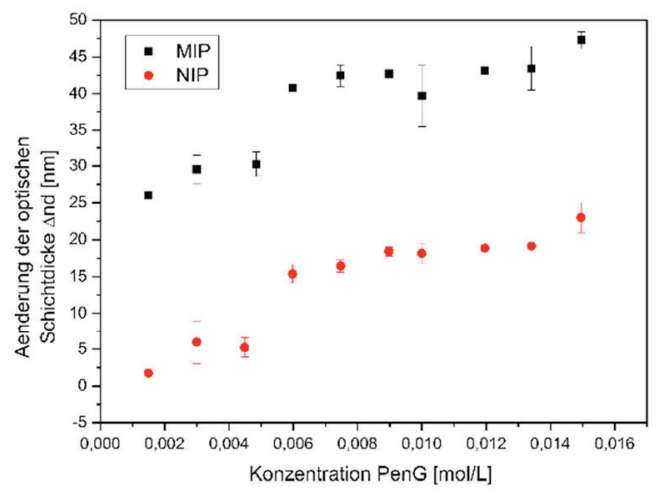

Abb.5: Dreifachmessungen verschiedener Konzentrationen. Die Konzentrationen liegen zwischen 1,49.10 ${ }^{3} \mathrm{~mol} / \mathrm{L}$ und $14,9 \cdot 10^{-3} \mathrm{~mol} / \mathrm{L}$. In rot sind die Ergebnisse des NIP-Spots und in schwarz die des MIP-Spots zu sehen.

\section{Diskussion und Ausblick}

Die MIP- und NIP-Partikel wurden erfolgreich durch elektrostatische Wechselwirkungen auf der Sensoroberfläche immobilisiert. Obwohl die Partikel nicht komplett homogen auf der Oberfläche immobilisiert wurden konnten (Abb.3), wurden erfolgreiche Messungen durchgeführt. Es konnten nicht nur die Sensorsignale der MIP- und NIP-Spot deutlich voneinander unterschieden (Abb.4) und somit referenziert werden, es konnten auch konzentrationsabhängige Dreifachmessungen durchgeführt werden (Abb.5). Der fast lineare Bereich $a b$ einer Konzentration von 5,98.10-3 mol/L, deutet darauf hin, dass auf der Sensoroberfläche eine Sättigung auftritt. Diese Sättigung führt dazu, dass keine konzentrationsabhängigen Signale mehr zu detektieren sind.

Um eine Kalibrierfunktion im dynamischen Bereich zu bestimmen, sollten weitere Messungen im Bereich unter $5,98 \cdot 10^{-3} \mathrm{~mol} / \mathrm{L}$ durchgeführt werden. Ein weiterer Schritt zur Etablierung des Sensors ist die Messungen von Proben in Realmedien.

\section{Literatur}

[1] R. Kessler, Prozessanalytik: Strategien und Fallbeispiele aus der industriellen Praxis, WILEY-VCH Verlag GmbH \& Co.KGaA, Weinheim, (2006), ISBN:3527-31196-3

[2] A. Brecht, G. Gauglitz, W. Nahm, Interferometric measurements used in chemical and biochemical sensors, Analysis 20, 135-140 (1992)

[3] H.-M. Schmitt, A. Brecht, J. Piehler, G. Gauglitz, An integrated system for optical biomolecular interaction analysis, Biosens. Bioelectron. 12, 809-816 (1997)

[4] J. Piehler, A. Brecht, G. Gauglitz, M. Zerlin, C. Maul, R. Thiericke, S. Grabley, Label-free monitoring of DNAligand interactions, Anal. Biochem. 249, 94-102 (1997); doi:10.1006/abio.1997.2160

[5] G. Gauglitz, Direct optical detection in bioanalysis: an update, Anal Bioanal Chem 398, 2363-2372 (2010); doi: 10.1007/s00216-010-3904-4

[6] M. Ewald, P. Fechner, G. Gauglitz, A multi-analyte biosensor for the simultaneous label-free detection of pathogens and biomarkers in point-of-need animal testing. Anal Bioanal Chem 407, 4005-4013, (2015), doi:10.1007/s00216-015-8562-0

\section{Danksagung}

Diese Arbeit ist im Rahmen des Projektes BioMIPBiomimetischer Sensor zur inline Prozessanalytik 
(FKZ 7533-7311.6-5A) des Landesministerium für Wissenschaft, Forschung und Kunst, BadenWürttemberg gefördert.

Wir danken Prof. Dr. Tovar und seiner Arbeitsgruppe für die Bereitstellung der Polymerpartikel. 